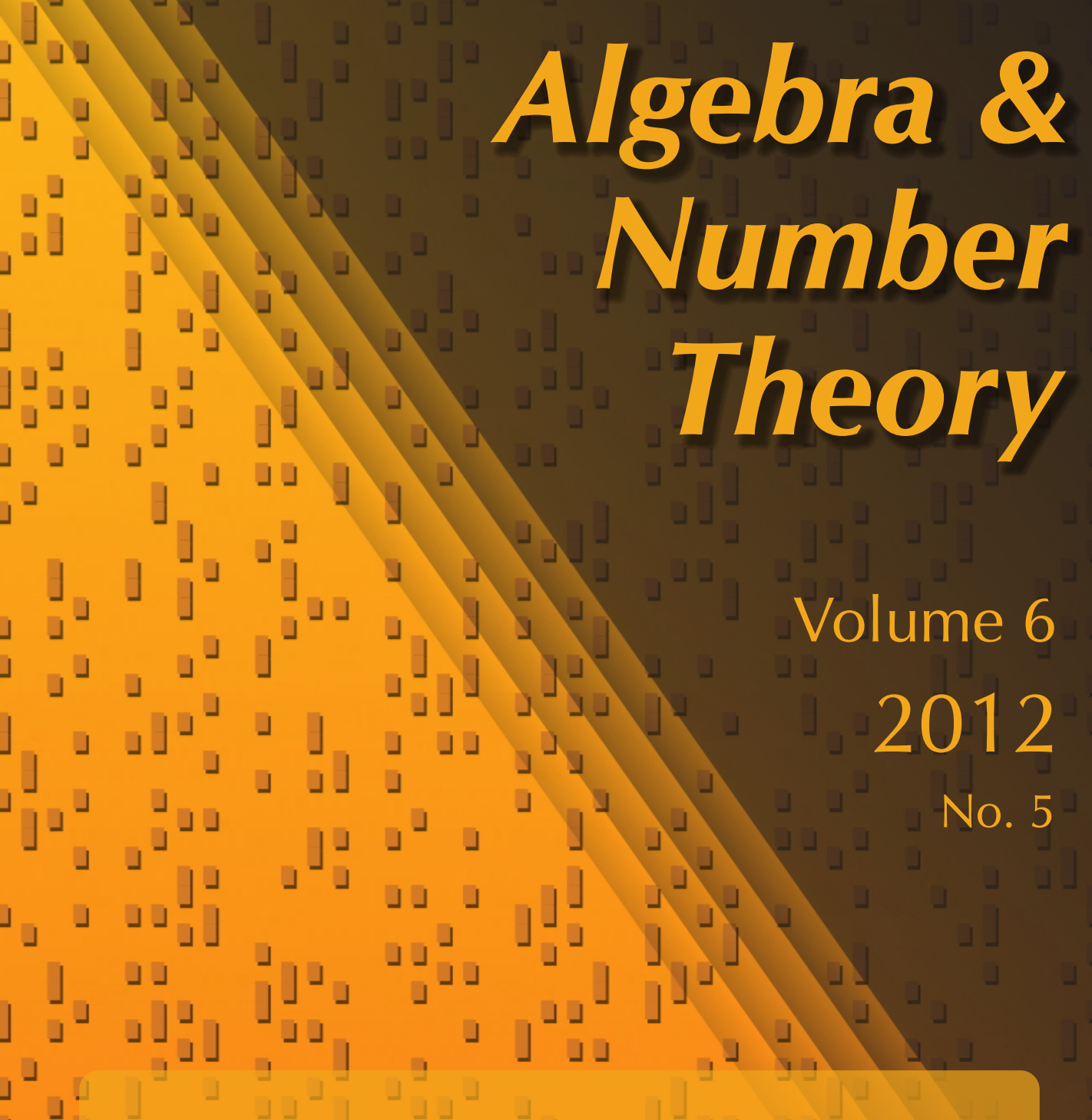

A denominator identity

for affine Lie superalgebras with zero dual Coxeter number

Maria Gorelik and Shifra Reif

\lrcorner

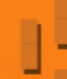

J

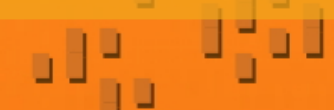

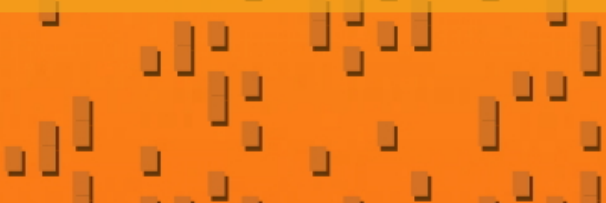

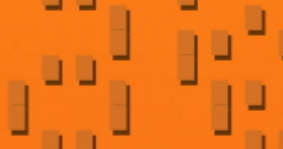

stithematical sciences pub hishers 


\title{
A denominator identity for affine Lie superalgebras with zero dual Coxeter number
}

\author{
Maria Gorelik and Shifra Reif
}

We prove a denominator identity for nontwisted affine Lie superalgebras with zero dual Coxeter number.

\section{Introduction}

0.1. Let $\mathfrak{g}$ be a complex finite-dimensional contragredient Lie superalgebra. These algebras were classified by V. Kac [1977] and the list (excluding Lie algebras) consists of four series: $A(m \mid n), B(m \mid n), C(m), D(m \mid n)$ and the exceptional algebras $D(2,1, a), F(4), G(3)$. The finite-dimensional contragredient Lie superalgebras with zero Killing form (or, equivalently, with dual Coxeter number equal to zero) are $A(n \mid n), D(n \mid n+1)$ and $D(2,1, a)$.

Denote by $\Delta_{+0}$ (resp., $\Delta_{+1}$ ) the set of positive even (resp., odd) roots of $\mathfrak{g}$. The Weyl denominator $R$ and the affine Weyl denominator $\hat{R}$ are given by the formulas

where

$$
R=\frac{R_{0}}{R_{1}}, \quad \hat{R}=\frac{\hat{R}_{0}}{\hat{R}_{1}}
$$

$$
\begin{array}{ll}
R_{0}:=\prod_{\alpha \in \Delta_{+0}}\left(1-e^{-\alpha}\right), & \hat{R}_{0}:=R_{0} \cdot \prod_{k=1}^{\infty}\left(1-q^{k}\right)^{\mathrm{rank} \mathfrak{g}} \prod_{\alpha \in \Delta_{0}}\left(1-q^{k} e^{-\alpha}\right), \\
R_{1}:=\prod_{\alpha \in \Delta_{+1}}\left(1+e^{-\alpha}\right), & \hat{R}_{1}:=R_{1} \cdot \prod_{k=1}^{\infty} \prod_{\alpha \in \Delta_{1}}\left(1+q^{k} e^{-\alpha}\right) .
\end{array}
$$

Let $\hat{\mathfrak{g}}$ be the nontwisted affinization of $\mathfrak{g}, \hat{\mathfrak{h}}$ be the Cartan subalgebra of $\hat{\mathfrak{g}}$ and $\hat{\Delta}_{+}$be the set of positive roots of $\hat{\mathfrak{g}}$. The affine Weyl denominator is the Weyl denominator of $\hat{\mathfrak{g}}$. Let $\hat{\rho} \in \hat{\mathfrak{h}}$ be such that $2(\hat{\rho}, \alpha)=(\alpha, \alpha)$ for each simple root $\alpha \in \hat{\Delta}_{+}$.

Supported in part by the Minerva Foundation with funding from the German Federal Ministry for Education and Research.

MSC2010: primary 17B67; secondary 17B22.

Keywords: Lie superalgebra, denominator identity. 
If $\mathfrak{g}$ has a nonzero Killing form, the affine denominator identity, stated in [Kac and Wakimoto 1994] and proven there and in [Gorelik 2011], takes the form

$$
\hat{R} e^{\hat{\rho}}=\sum_{w \in T^{\prime}} w\left(R e^{\hat{\rho}}\right),
$$

where $T^{\prime}$ is the affine translation group corresponding to the "largest" root subsystem of $\Delta_{0}$. The affine denominator identity for strange Lie superalgebras $Q(n)$, which are not contragredient, was stated in [Kac and Wakimoto 1994] and proven in [Zagier 2000].

For a parameter $q$ and a formal variable $x$ we introduce, after [De Sole and Kac 2005], the infinite products

$$
(1+x)_{q}^{\infty}:=\prod_{k=0}^{\infty}\left(1+q^{k} x\right) \quad \text { and } \quad(1-x)_{q}^{\infty}:=\prod_{k=0}^{\infty}\left(1-q^{k} x\right) .
$$

These infinite products converge for any $x \in \mathbb{C}$ if the parameter $q$ is a real number $0<q<1$. In particular, they are well defined for $0<x=q<1$ and $(1 \pm q)_{q}^{\infty}:=$ $\prod_{n=1}^{\infty}\left(1 \pm q^{n}\right)$.

For $A(n-1 \mid n-1)=\mathfrak{g l}(n \mid n)$ denote by $\mathfrak{s t r}$ the restriction of the supertrace to the Cartan subalgebra $\mathfrak{h} \subset \mathfrak{g}$ (thus $\mathfrak{s t r} \in \mathfrak{h}^{*}$ ).

In this paper we will prove the following theorem.

0.2. Theorem. Let $\mathfrak{g}$ be a complex finite-dimensional contragredient Lie superalgebra with zero Killing form. One has

$$
\begin{array}{ll}
\hat{R} e^{\hat{\rho}} \cdot f\left(q, e^{\mathfrak{s t r}}\right)=\sum_{w \in T^{\prime}} w\left(\operatorname{Re}^{\hat{\rho}}\right) & \text { for } A(n \mid n), \\
\hat{R} e^{\hat{\rho}} \cdot f(q)=\sum_{w \in T^{\prime}} w\left(\operatorname{Re}^{\hat{\rho}}\right) & \text { for } D(n+1 \mid n), D(2,1, a),
\end{array}
$$

where $T^{\prime}$ is the affine translation group corresponding to the "smallest" root subsystem of $\Delta_{0}$ (see 0.4 below) and $f\left(q, e^{\text {str }}\right), f(q)$ are given by the following formulas

$$
\begin{array}{ll}
f\left(q, e^{\mathfrak{s t r}}\right)=\frac{\left(1-q(-1)^{n} e^{\mathfrak{s t r}}\right)_{q}^{\infty} \cdot\left(1-q(-1)^{n} e^{-\mathfrak{s t r}}\right)_{q}^{\infty}}{\left((1-q)_{q}^{\infty}\right)^{2}} & \text { for } \mathfrak{g l}(n \mid n), \\
f(q)=\left((1-q)_{q}^{\infty}\right)^{-1} & \text { for } D(n+1 \mid n) .
\end{array}
$$

0.3. The affine denominator identity for $\mathfrak{g l}(2 \mid 2)$ was stated by V. Kac and M. Wakimoto [1994] and proven in [Gorelik 2010] (with a proof different from the one presented below).

As pointed by P. Etingof, the terms $f\left(q, e^{\mathfrak{s t r}}\right), f(q)$ can be interpreted using "degenerate" cases $n=1$; for example, for $\mathfrak{g l}(1 \mid 1)$ we obtain the formula

$$
\hat{R} e^{\hat{\rho}}=\frac{\left((1-q)_{q}^{\infty}\right)^{2}}{\left(1+q e^{\mathfrak{s t r}}\right)_{q}^{\infty} \cdot\left(1+q e^{-\mathfrak{s t r}}\right)_{q}^{\infty}} R e^{\hat{\rho}}
$$


which is trivial since $\mathfrak{g l}(1 \mid 1)$ has the only positive $\operatorname{root} \beta=\mathfrak{s t r}$, which is odd.

Since $\mathfrak{s l}(n \mid n)=\{a \in \mathfrak{g l}(n \mid n): \mathfrak{s t r}(a)=0\}$ and

$$
\operatorname{rank} \mathfrak{s l}(n \mid n)=2 n-1=\operatorname{rank} \mathfrak{g l}(n \mid n)-1,
$$

one has

$$
f(q)=\left\{\begin{array}{cl}
(1-q)_{q}^{\infty} & \text { for } \mathfrak{s l}(2 n \mid 2 n), \\
\frac{\left((1+q)_{q}^{\infty}\right)^{2}}{(1-q)_{q}^{\infty}} & \text { for } \mathfrak{s l}(2 n+1 \mid 2 n+1) .
\end{array}\right.
$$

The root datum of $D(2,1, a)$ is the same as the root datum of $D(2 \mid 1)$ so the affine denominator identity for $D(2,1, a)$ is the same as the affine denominator identity for $D(2 \mid 1)$.

As it is shown in [Kac and Wakimoto 1994], the evaluation of the affine denominator identity (2) for $A(1 \mid 1)$ gives the following Jacobi identity [1829]:

$$
\square(q)^{8}=1+16 \sum_{j, k=1}^{\infty}(-1)^{(j+1) k} k^{3} q^{j k},
$$

where $\square(q)=\sum_{j \in \mathbb{Z}} q^{j^{2}}$ and thus the coefficient of $q^{m}$ in the power series expansion of $\square(q)^{8}$ is the number of representation of a given integer as a sum of 8 squares (taking into the account the order of summands).

0.4. In order to define $T^{\prime}$ for $A(n \mid n), D(n+1 \mid n)$ we present the set of even roots in the form $\Delta_{0}=\Delta^{\prime} \amalg \Delta^{\prime \prime}$, where

$$
\begin{array}{ll}
\Delta^{\prime} \cong \Delta^{\prime \prime}=A_{n-1} & \text { for } A(n-1 \mid n-1)=\mathfrak{g l}(n \mid n), \\
\Delta^{\prime}=C_{n}, \Delta^{\prime \prime}=D_{n+1} & \text { for } D(n+1 \mid n) .
\end{array}
$$

Let $W^{\prime}$ be the Weyl group of $\Delta^{\prime}$ and $\hat{W}^{\prime}$ be the corresponding affine Weyl group. Then $\hat{W}^{\prime}=W^{\prime} \ltimes T^{\prime}$, where $T^{\prime}$ is a translation group, see [Kac 1990, Chapter VI]. By contrast to Lie superalgebras with nonzero Killing form, for $D(n+1 \mid n)$ the rank of root system $\Delta^{\prime}$ is smaller than the rank of $\Delta^{\prime \prime}$. It is not possible to change $T^{\prime}$ to $T^{\prime \prime}$ in (1) and in (2) for $D(n+1 \mid n)$, since the sum $\sum_{w \in T^{\prime \prime}} w\left(R e^{\hat{\rho}}\right)$ is not well defined if $\Delta^{\prime} \not \Delta^{\prime \prime}$ (see Remark 2.1.4).

The key point of our proof of Theorem 0.2 is Proposition 2.3.2, where it is shown that the expansion of $Y:=\hat{R}^{-1} e^{-\hat{\rho}} \sum_{w \in T^{\prime}} w\left(\operatorname{Re}^{\hat{\rho}}\right)$ contains only $\hat{W}$-invariant elements. This implies that $Y=f(q)$ for $\mathfrak{g}=D(n+1 \mid n)$ and $Y=f\left(q, e^{-\mathfrak{s t r}}\right)$ for $\mathfrak{g l}(n \mid n)$. We determine $f(q)$ and $f\left(q, e^{\mathfrak{s t r}}\right)$ using suitable evaluations.

\section{Preliminaries}

One readily sees (for instance, [Gorelik 2011, 1.5]) that $R e^{\hat{\rho}}$ and $\hat{R} e^{\hat{\rho}}$ do not depend on the choice of set of positive roots $\Delta_{+}$. As a result, in order to prove Theorem 0.2 , 
it is enough to establish the identity (2) for one choice of $\Delta_{+}$. Similarly, it is enough to establish the identity for one choice of $A_{n-1}$ for $\mathfrak{g l}(n \mid n)$. In Section 1.1 we describe our choice of the set of positive roots for $\mathfrak{g l}(n \mid n), D(n+1 \mid n)$. In Section 1.2 we introduce notation for affine Lie superalgebra $\hat{\mathfrak{g}}$. In Section 1.3 we introduce the algebra $\mathscr{R}$ of formal power series in which we expand $R$ and $\hat{R}$.

Note that if the dual Coxeter number of $\mathfrak{g}$ is zero, then

$$
\hat{\rho}=\rho=\frac{1}{2}\left(\sum_{\alpha \in \Delta_{+0}} \alpha-\sum_{\alpha \in \Delta_{+1}} \alpha\right) .
$$

1.1. Root systems. Let $\mathfrak{g}$ be $\mathfrak{g l}(n \mid n)$ or $D(n \mid n+1)$ and let $\mathfrak{h}$ be its Cartan subalgebra. We fix the following sets of simple roots:

$$
\Pi= \begin{cases}\left\{\varepsilon_{1}-\delta_{1}, \delta_{1}-\varepsilon_{2}, \varepsilon_{2}-\delta_{2}, \ldots, \varepsilon_{n}-\delta_{n}\right\} & \text { for } \mathfrak{g l}(n \mid n), \\ \left\{\varepsilon_{1}-\delta_{1}, \delta_{1}-\varepsilon_{2}, \varepsilon_{2}-\delta_{2}, \ldots, \varepsilon_{n}-\delta_{n}, \delta_{n} \pm \varepsilon_{n+1}\right\} & \text { for } D(n+1 \mid n) .\end{cases}
$$

We fix a nondegenerate symmetric invariant bilinear form on $\mathfrak{g}$ and denote by $(-,-)$ the induced nondegenerate symmetric bilinear form on $\mathfrak{h}^{*}$; we normalize the form in such a way that $-\left(\varepsilon_{i}, \varepsilon_{j}\right)=\left(\delta_{i}, \delta_{j}\right)=\delta_{i j}$; notice that $\left\{\varepsilon_{i}, \delta_{i}: 1 \leq i \leq n\right\}$ (resp., $\left\{\varepsilon_{j}, \delta_{i}: 1 \leq i \leq n, 1 \leq j \leq n+1\right\}$ ) is an orthogonal basis of $\mathfrak{h}^{*}$ for $\mathfrak{g l}(n \mid n)$ (resp., for $D(n+1 \mid n))$.

For this choice one has

$$
\begin{gathered}
\Delta_{0+}= \begin{cases}\left\{\varepsilon_{i}-\varepsilon_{j}\right\}_{1 \leq i<j \leq n} \amalg\left\{\delta_{i}-\delta_{j}\right\}_{1 \leq i<j \leq n} & \text { for } \mathfrak{g l}(n \mid n), \\
\Delta_{0+}=\left\{\varepsilon_{i} \pm \varepsilon_{j}\right\}_{1 \leq i<j \leq n+1} \amalg\left\{\delta_{s} \pm \delta_{t}\right\}_{1 \leq s<t \leq n} \cup\left\{2 \delta_{s}\right\}_{1 \leq s \leq n} & \text { for } D(n+1 \mid n),\end{cases} \\
\Delta_{1+}= \begin{cases}\left\{\varepsilon_{i}-\delta_{j}\right\}_{1 \leq i \leq j \leq n} \cup\left\{\delta_{i}-\varepsilon_{j}\right\}_{1 \leq i<j \leq n} & \text { for } \mathfrak{g l}(n \mid n), \\
\Delta_{1+}=\left\{\varepsilon_{i}-\delta_{s}\right\}_{1 \leq i \leq s \leq n} \cup\left\{\delta_{s}-\varepsilon_{j}\right\}_{1 \leq s<j \leq n+1} \cup\left\{\delta_{i}+\varepsilon_{j}\right\}_{1 \leq i \leq n ; 1 \leq j \leq n+1} & \text { for } D(n+1 \mid n) .\end{cases}
\end{gathered}
$$

For $D(n+1 \mid n)$ one has $\rho=0$. For $\mathfrak{g l}(n \mid n)$ one has $\mathfrak{s t r}=\sum_{i=1}^{n}\left(\varepsilon_{i}-\delta_{i}\right)$ and $\rho=-\frac{1}{2} \mathfrak{s t r}$.

Recall that $\mathfrak{s l}(n \mid n)=\{a \in \mathfrak{g l}(n \mid n): \mathfrak{s t r}(a)=0\}$ and so $\mathfrak{h}^{*}$ for $\mathfrak{s l}(n \mid n)$ is the quotient of $\mathfrak{h}^{*}$ for $\mathfrak{g l}(n \mid n)$ by $\mathbb{C} \mathfrak{s t r}$.

By the above, $\Delta_{0}$ is the union of two irreducible root systems, and we write $\Delta_{0}=\Delta^{\prime \prime} \amalg \Delta^{\prime}$, where $\Delta^{\prime \prime}$ lies in the span of the $\varepsilon_{i}$ and $\Delta^{\prime}$ lies in the span of the $\delta_{i}$ (this notation is compatible with the notation in Section 0.4).

1.2. Nontwisted affinization. Let $\mathfrak{g}=\mathfrak{n}_{-} \oplus \mathfrak{h} \oplus \mathfrak{n}_{+}$be any complex finite-dimensional contragredient Lie superalgebra with a fixed triangular decomposition, and let $\Delta_{+}$ be its set of positive roots. Let $\hat{\mathfrak{g}}$ be the affinization of $\mathfrak{g}$ and let $\hat{\mathfrak{h}}$ be its Cartan subalgebra, see [Kac 1990, Chapter VI]. Let $\hat{\Delta}=\hat{\Delta}_{0} \amalg \hat{\Delta}_{1}$ be the set of roots of $\hat{\mathfrak{g}}$. We set

$$
\hat{\Delta}^{+}=\Delta_{+} \cup\left(\bigcup_{k=1}^{\infty}\{\alpha+k \delta \mid \alpha \in \Delta\}\right) \cup\left(\bigcup_{k=1}^{\infty}\{k \delta\}\right),
$$


where $\delta$ is the minimal imaginary root. Let $W$ and $\hat{W}$ be the Weyl groups of $\Delta_{0}$ and $\hat{\Delta}_{0}$. One has $\left(\hat{\mathfrak{h}}^{*}\right)^{\hat{W}}=\mathbb{C} \delta$ for $\mathfrak{g} \neq \mathfrak{g l}(n \mid n)$ and $\left(\hat{\mathfrak{h}}^{*}\right)^{\hat{W}}=\mathbb{C} \delta \oplus \mathbb{C} \mathfrak{s t r}$ for $\mathfrak{g}=\mathfrak{g l}(n \mid n)$.

We extend the nondegenerate symmetric invariant bilinear form from $\mathfrak{g}$ to $\hat{\mathfrak{g}}$ and denote by $(-,-)$ the induced nondegenerate symmetric bilinear form on $\hat{\mathfrak{h}}^{*}$ (the above-mentioned form on $\mathfrak{h}^{*}$ is induced by this form on $\hat{\mathfrak{h}}^{*}$ ). For $A \subset \hat{\mathfrak{h}}^{*}$ we set $A^{\perp}=\left\{\mu \in \hat{\mathfrak{h}}^{*}: \forall v \in A,(\mu, v)=0\right\}$.

1.2.1. In Section 1.1 we introduced the root systems $\Delta^{\prime}, \Delta^{\prime \prime}$ for $\mathfrak{g}=\mathfrak{g l}(n \mid n)$ and $\mathfrak{g}=D(n+1 \mid n)$. Let $W^{\prime}$ and $W^{\prime \prime}$ be the Weyl groups of $\Delta^{\prime}$ and $\Delta^{\prime \prime}$, respectively. One has $W=W^{\prime} \times W^{\prime \prime}$. We denote by $\hat{W}^{\prime}$ the Weyl group of the affine root system $\hat{\Delta}^{\prime}$. Recall that $\hat{W}^{\prime}=W^{\prime} \ltimes T^{\prime}$, where $T^{\prime}$ is a translation group; see [Kac 1990, Chapter VI].

1.2.2. For $N \subset \hat{\mathfrak{h}}^{*}$ we use the notation $\mathbb{Z} N$ for the set $\sum_{\mu \in N} \mathbb{Z} \mu$. Set

$$
Q^{+}:=\sum_{\mu \in \Delta_{+}} \mathbb{Z}_{\geq 0} \mu, \quad Q:=\mathbb{Z} \Delta_{+}, \quad \hat{Q}^{ \pm}:= \pm \sum_{\mu \in \hat{\Delta}_{+}} \mathbb{Z}_{\geq 0} \mu, \quad \hat{Q}:=\mathbb{Z} \hat{\Delta}_{+} .
$$

We introduce the standard partial order on $\hat{\mathfrak{h}}^{*}: \mu \leq v$ if $(v-\mu) \in \hat{Q}^{+}$.

1.3. The algebra $\mathscr{R}$. We are going to use the notation of [Gorelik 2011, 1.4], which we recall below. We retain the notation of Section 1.2.

1.3.1. Call a $\hat{Q}^{+}$-cone a set of the form $\left(\lambda-\hat{Q}^{+}\right)$, where $\lambda \in \hat{\mathfrak{h}}^{*}$.

For a formal sum of the form $Y:=\sum_{\nu \in \hat{h}^{*}} b_{\nu} e^{\nu}, b_{v} \in \mathbb{Q}$ define the support of $Y$ by $\operatorname{supp}(Y):=\left\{v \in \hat{\mathfrak{h}}^{*}: b_{v} \neq 0\right\}$. Let $\mathscr{R}$ be a vector space over $\mathbb{Q}$, spanned by the sums of the form $\sum_{v \in \hat{Q}^{+}} b_{\nu} e^{\lambda-v}$, where $\lambda \in \hat{\mathfrak{h}}^{*}, b_{v} \in \mathbb{Q}$. In other words, $\mathscr{R}$ consists of the formal sums $Y=\sum_{v \in \hat{\mathfrak{h}}^{*}} b_{\nu} e^{v}$ with the support lying in a finite union of $\hat{Q}^{+}$-cones.

Clearly, $\mathscr{R}$ has a structure of commutative algebra over $\mathbb{Q}$. If $Y \in \mathscr{R}$ is such that $Y Y^{\prime}=1$ for some $Y^{\prime} \in \mathscr{R}$, we write $Y^{-1}:=Y^{\prime}$.

1.3.2. Action of the Weyl group. For $w \in \hat{W}$ set $w\left(\sum_{v \in \hat{\mathfrak{h}}^{*}} b_{\nu} e^{v}\right):=\sum_{v \in \hat{\mathfrak{h}}^{*}} b_{\nu} e^{w v}$. By the above, $w Y \in \mathscr{R}$ if and only if $w(\operatorname{supp} Y)$ is a subset of a finite union of $\hat{Q}^{+}$. cones. For each subgroup $\tilde{W}$ of $\hat{W}$ we set $\mathscr{R}_{\tilde{W}}:=\{Y \in \mathscr{R}: w Y \in \mathscr{R}$ for each $w \in \tilde{W}\}$; notice that $\mathscr{R}_{\tilde{W}}$ is a subalgebra of $\mathscr{R}$.

1.3.3. Infinite products. An infinite product of the form $Y=\prod_{\nu \in X}\left(1+a_{\nu} e^{-v}\right)^{r(v)}$, where $a_{v} \in \mathbb{Q}, \quad r(v) \in \mathbb{Z}_{\geq 0}$ and $X \subset \hat{\Delta}$ is such that the set $X \backslash \hat{\Delta}_{+}$is finite, can be naturally viewed as an element of $\mathscr{R}$; clearly, this element does not depend on the order of factors. Let $y$ be the set of such infinite products. For any $w \in \hat{W}$ the infinite product

$$
w Y:=\prod_{v \in X}\left(1+a_{\nu} e^{-w v}\right)^{r(v)},
$$


is again an infinite product of the above form, since the set $w \hat{\Delta}_{+} \backslash \hat{\Delta}_{+}$is finite (see for example [Gorelik 2011, Lemma 1.2.8]). Hence $y$ is a $\hat{W}$-invariant multiplicative subset of $\mathscr{R}_{\hat{W}}$.

The elements of $\mathscr{Y}$ are invertible in $\mathscr{R}$ : using the geometric series we can expand $Y^{-1}$. For example, $\left(1-e^{\alpha}\right)^{-1}=-e^{-\alpha}\left(1-e^{-\alpha}\right)^{-1}=-\sum_{i=1}^{\infty} e^{-i \alpha}$.

1.3.4. The subalgebra $\mathscr{R}^{\prime}$. Denote by $\mathscr{R}^{\prime}$ the localization of $\mathscr{R}_{\hat{W}}$ by $\mathscr{Y}$. By the above, $\mathscr{R}^{\prime}$ is a subalgebra of $\mathscr{R}$. Observe that $\mathscr{R}^{\prime} \not \subset \mathscr{R}_{\hat{W}}$ : for example, $\left(1-e^{-\alpha}\right)^{-1} \in \mathscr{R}^{\prime}$, but $\left(1-e^{-\alpha}\right)^{-1}=\sum_{j=0}^{\infty} e^{-j \alpha} \notin \mathscr{R}_{\hat{W}}$. We extend the action of $\hat{W}$ from $\mathscr{R}_{\hat{W}}$ to $\mathscr{R}^{\prime}$ by setting $w\left(Y^{-1} Y^{\prime}\right):=(w Y)^{-1}\left(w Y^{\prime}\right)$ for $Y \in \mathscr{Y}, Y^{\prime} \in \mathscr{R}_{\hat{W}}$.

Notice that an infinite product of the form $Y=\prod_{\nu \in X}\left(1+a_{\nu} e^{-v}\right)^{r(v)}$, where $a_{v}, X$ are as above and $r(v) \in \mathbb{Z}$, lies in $\mathscr{R}^{\prime}$ and $w Y=\prod_{v \in X}\left(1+a_{v} e^{-w v}\right)^{r(v)}$. The $\operatorname{support} \operatorname{supp}(Y)$ has a unique maximal element (with respect to the standard partial order) and this element is given by the formula

$$
\max \operatorname{supp}(Y)=-\sum_{\nu \in X \backslash \hat{\Delta}_{+}: a_{\nu} \neq 0} r_{\nu} v .
$$

1.3.5. Let $\tilde{W}$ be a subgroup of $\hat{W}$. For $Y \in \mathscr{R}^{\prime}$ we say that $Y$ is $\tilde{W}$-invariant (resp., $\tilde{W}$-anti-invariant) if $w Y=Y($ resp., $w Y=\operatorname{sgn}(w) Y)$ for each $w \in \tilde{W}$.

Let $Y=\sum a_{\mu} e^{\mu} \in \mathscr{R}_{\tilde{W}}$ be $\tilde{W}$-anti-invariant. Then $a_{w \mu}=(-1)^{\operatorname{sgn}(w)} a_{\mu}$ for each $\mu$ and $w \in \tilde{W}$. In particular, $\tilde{W} \operatorname{supp}(Y)=\operatorname{supp}(Y)$, and, moreover, for each $\mu \in \operatorname{supp}(Y)$ one has $\operatorname{Stab}_{\tilde{W}} \mu \subset\{w \in \tilde{W}: \operatorname{sgn}(w)=1\}$. The condition $Y \in \mathscr{R}_{\tilde{W}}$ is essential: for example, for $\tilde{W}=\left\{\mathrm{id}, s_{\alpha}\right\}$, the expressions $Y:=e^{\alpha}-e^{-\alpha}$, $Y^{-1}=e^{-\alpha}\left(1-e^{-2 \alpha}\right)^{-1}$ are $\tilde{W}$-anti-invariant, $\operatorname{supp}(Y)=\{ \pm \alpha\}$ is $s_{\alpha}$-invariant, but $\operatorname{supp}\left(Y^{-1}\right)=\{-\alpha,-3 \alpha, \ldots\}$ is not $s_{\alpha}$-invariant.

For $Y \in \mathscr{R}_{\tilde{W}}$ such that each $\tilde{W}$-orbit in $\hat{\mathfrak{h}}^{*}$ has a finite intersection with $\operatorname{supp}(Y)$, introduce the sum

$$
\mathscr{F}_{\tilde{W}}(Y):=\sum_{w \in \tilde{W}} \operatorname{sgn}(w) w Y .
$$

This sum is well defined, but does not always belong to $\mathscr{R}$. For $Y=\sum a_{\mu} e^{\mu}$ one has $\mathscr{F}_{\tilde{W}}(Y)=\sum b_{\mu} e^{\mu}$, where $b_{\mu}=\sum_{w \in \tilde{W}} \operatorname{sgn}(w) a_{w \mu}$; in particular, $b_{\mu}=\operatorname{sgn}(w) b_{w \mu}$ for each $w \in \tilde{W}$. One has

$$
Y \in \mathscr{R}_{\tilde{W}} \text { and } \mathscr{F}_{\tilde{W}}(Y) \in \mathscr{R} \Longrightarrow\left\{\begin{array}{l}
\mathscr{F}_{\tilde{W}}(Y) \in \mathscr{R}_{\tilde{W}}, \\
\operatorname{supp}\left(\mathscr{F}_{\tilde{W}}(Y)\right) \text { is } \tilde{W} \text {-stable } \\
\mathscr{F}_{\tilde{W}}(Y) \text { is } \tilde{W} \text {-anti-invariant. }
\end{array}\right.
$$

We call a vector $\lambda \in \hat{\mathfrak{h}}^{*} \tilde{W}$-regular if $\operatorname{Stab}_{\tilde{W}} \lambda=\{\mathrm{id}\}$, and we say that the orbit $\tilde{W} \lambda$ is $\tilde{W}$-regular if $\lambda$ is $\tilde{W}$-regular (so the orbit consists of $\tilde{W}$-regular points). If $\tilde{W}$ is an affine Weyl group, then for any $\lambda \in \hat{\mathfrak{h}}^{*}$ the stabilizer $\operatorname{Stab}_{\tilde{W}} \lambda$ is either trivial 
or contains a reflection. Thus for $\tilde{W}=\hat{W}^{\prime}, \hat{W}^{\prime \prime}$ one has

$Y \in \mathscr{R}_{\tilde{W}}$ and $\mathscr{F}_{\tilde{W}}(Y) \in \mathscr{R} \Longrightarrow \operatorname{supp}\left(\mathscr{F}_{\tilde{W}}(Y)\right)$ is a union of $\tilde{W}$-regular orbits.

\section{Proof}

Unless stated otherwise, $\mathfrak{g}$ is assumed to be one of the algebras $\mathfrak{g l}(n \mid n), D(n+1 \mid n)$.

As it is pointed out in Section 1, it is enough to establish the denominator identity for a particular choice of $\Delta_{+}$and we do this for the choice described in Section 1.1. Recall that the group $T^{\prime}$ was introduced in Section 1.2.1. The steps of the proof are the following.

- In Section 2.1 we check that the sum $\mathscr{F} T^{\prime}\left(R e^{\hat{\rho}}\right)$ is well-defined and belongs to $\mathscr{R}$.

- In Section 2.2 we prove the inclusions

$$
\operatorname{supp}\left(\mathscr{F}_{T^{\prime}}\left(R e^{\hat{\rho}}\right)\right), \operatorname{supp}\left(\hat{R} e^{\hat{\rho}}\right) \subset U,
$$

where

$$
U:=\left\{\mu \in \hat{\rho}-\hat{Q}^{+}:(\mu, \mu)=(\hat{\rho}, \hat{\rho})\right\} .
$$

We remark that (5) holds for simple contragredient Lie superalgebras with nonzero Killing form; see [Gorelik 2011, 2.4].

- In Section 2.3 we show that if the dual Coxeter number of $\mathfrak{g}$ is zero, then the inclusions (5) imply that $\operatorname{supp}\left(\hat{R}^{-1} e^{-\hat{\rho} \mathscr{F}} T^{\prime}\left(R e^{\hat{\rho}}\right)\right) \subset \hat{Q}^{\hat{W}}$. As a result, $\hat{R}^{-1} e^{-\hat{\rho} \mathscr{F}} T^{\prime}\left(R e^{\hat{\rho}}\right)$ takes the form $f(q)$ for $\mathfrak{g} \neq \mathfrak{g l}(n \mid n)$ and $f\left(q, e^{\mathfrak{s t r}}\right)$ for $\mathfrak{g l}(n \mid n)$.

- In Section 2.4 we compute $f(q)$ for $D(n+1 \mid n)$ and $f\left(q, e^{\mathfrak{s t r}}\right)$ for $\mathfrak{g l}(n \mid n)$. This completes the proof of the identities (2).

2.1. In this subsection we show that for $\mathfrak{g}=\mathfrak{g l}(n \mid n), D(n+1 \mid n)$, the sum $\mathscr{F}_{T^{\prime}}\left(\operatorname{Re}^{\hat{\rho}}\right)$ is a well-defined element of $\mathscr{R}$. Since $\hat{\rho}=\rho$ is $\hat{W}$-invariant, it is enough to verify that $\mathscr{F}_{T^{\prime}}(R)$ is a well-defined element of $\mathscr{R}$. where

Recall that $T^{\prime}=\mathbb{Z}\left\{t_{\delta_{i}-\delta_{i+1}}\right\}_{i=1}^{n-1}$ for $\mathfrak{g l}(n \mid n)$ and $T^{\prime}=\mathbb{Z}\left\{t_{\delta_{i}}\right\}_{i=1}^{n}$ for $D(n+1 \mid n)$,

$$
t_{\mu}(\alpha)=\alpha-(\alpha, \mu) \delta \text { for any } \alpha \in \hat{Q} .
$$

2.1.1. By Section 1.3.4 one has

$$
\max \operatorname{supp}(w(R))=-\sum_{\substack{\alpha \in \Delta_{0+}: \\ w \alpha<0}} w \alpha+\sum_{\substack{\beta \in \Delta_{1+} \\ w \beta<0}} w \beta .
$$

For $w \in T^{\prime}$ write $w=t_{\mu}$, where $\mu \in \mathbb{Z}\left\{\delta_{i}-\delta_{i+1}\right\}_{1 \leq i<n}$ for $\mathfrak{g l}(n \mid n)$ and $\mu \in \mathbb{Z}\left\{\delta_{i}\right\}_{i=1}^{n}$ for $D(n+1 \mid n)$. From (7) we get

$$
\left\{\beta \in \Delta_{i+} \mid w \beta<0\right\}=\left\{\beta \in \Delta_{i+} \mid(\beta, \mu)>0\right\} \text { for } i=0,1 .
$$


We obtain $\max \operatorname{supp}\left(t_{\mu}(R)\right)=-v(\mu)+(v(\mu), \mu) \delta$, where

$$
v(\mu):=\sum_{\substack{\beta \in \Delta_{0+}: \\(\beta, \mu)>0}} \beta-\sum_{\substack{\beta \in \Delta_{1+}: \\(\beta, \mu)>0}} \beta .
$$

In order to prove that $\mathscr{F}_{T^{\prime}}(R)$ is a well-defined element of $\mathscr{R}$ we verify that

(i) $(v(\mu), \mu) \leq 0 \quad$ for all $\mu$;

(ii) $\{\mu:(v(\mu), \mu) \geq-N\}$ is finite for all $N>0$.

Condition (ii) ensures that the sum $\mathscr{F}_{F} T^{\prime}(R)=\sum_{\mu} t_{\mu}(R)$ is well-defined and condition (i) means that for each $\mu$ one has

$$
\max \operatorname{supp}\left(t_{\mu}(R)\right)=-v(\mu) \leq \sum_{\beta \in \Delta_{1+}} \beta
$$

so $\operatorname{supp}\left(\mathscr{F}_{T^{\prime}}(R)\right) \subset \sum_{\beta \in \Delta_{1+}} \beta-\hat{Q}^{+}$and thus $\mathscr{F}_{T^{\prime}}(R) \in \mathscr{R}$.

2.1.2. Case $\mathfrak{g l}(n \mid n)$. Recall that $w \in T^{\prime}$ has the form $w=t_{\mu}, \mu=\sum_{i=1}^{n} k_{i} \delta_{i}$, where the $k_{i} \mathrm{~s}$ are integers and $\sum_{i=1}^{n} k_{i}=0$. One has

$$
\begin{aligned}
& \left\{\alpha \in \Delta_{+0}:(\alpha, \mu)>0\right\}=\left\{\delta_{i}-\delta_{j}: i<j, k_{i}>k_{j}\right\}, \\
& \left\{\alpha \in \Delta_{+1}:(\alpha, \mu)>0\right\}=\left\{\varepsilon_{i}-\delta_{j}: k_{j}<0, i \leq j\right\} \cup\left\{\delta_{i}-\varepsilon_{j}: k_{i}>0, i<j\right\},
\end{aligned}
$$

where $1 \leq i, j \leq n$.

Write $v(\mu)=v^{\prime}+v^{\prime \prime}$, where $v^{\prime}=\sum_{i=1}^{n} a_{i} \delta_{i}$ and $v^{\prime \prime}$ lies in the span of the $\varepsilon_{i}$. By the above, for $k_{i}>0$ one has $a_{i} \leq(n-i)-(n-i)=0$ and for $k_{j}<0$ one has $a_{j} \geq-(j-1)+j=1$. Therefore

$$
(v(\mu), \mu)=\sum_{i=1}^{n} a_{i} k_{i} \leq \sum_{k_{i}<0} k_{i} \leq 0
$$

and the set $\{\mu:(v(\mu), \mu) \geq-N\}$ is a subset of the set $\left\{\mu: \sum_{k_{i}<0} k_{i} \geq-N\right\}$, which is finite for any $N$, because the $k_{i}$ are integers and $\sum_{i=1}^{n} k_{i}=0$. This establishes conditions (8).

2.1.3. Case $D(n+1 \mid n)$. Recall that $w \in T^{\prime}$ has the form $w=t_{\mu}, \mu=\sum k_{i} \delta_{i}$, where the $k_{i}$ s are integers. One has

$\left\{\alpha \in \Delta_{+0}:(\alpha, \mu)>0\right\}=$

$$
\left\{\delta_{i}-\delta_{j}: i<j, k_{i}>k_{j}\right\} \cup\left\{\delta_{i}+\delta_{j}: i \neq j, k_{i}+k_{j}>0\right\} \cup\left\{2 \delta_{i}: k_{i}>0\right\},
$$

$\left\{\alpha \in \Delta_{+1}:(\alpha, \mu)>0\right\}=$

$$
\left\{\varepsilon_{s}-\delta_{j}: k_{j}<0, s \leq j\right\} \cup\left\{\delta_{i}-\varepsilon_{s}: k_{i}>0, i<s\right\} \cup\left\{\delta_{i}+\varepsilon_{s}: k_{i}>0\right\},
$$

where $1 \leq i, j \leq n$ and $1 \leq s \leq n+1$. 
Write $v(\mu)=v^{\prime}+v^{\prime \prime}$, where $v^{\prime}=\sum_{i=1}^{n} a_{i} \delta_{i}$ and $v^{\prime \prime}$ lies in the span of the $\varepsilon_{i}$. By the above, for $k_{i}>0$ one has $a_{i} \leq(2 n+1-i)-(2 n+2-i)=-1$ and for $k_{j}<0$ one has $a_{j} \geq-(j-1)+j=1$. Therefore

$$
(v(\mu), \mu)=\sum_{i=1}^{n} a_{i} k_{i} \leq-\sum_{k_{i}>0} k_{i}+\sum_{k_{j}<0} k_{j}=-\sum_{1=1}^{n}\left|k_{i}\right| \leq 0,
$$

so the set $\{\mu:(v(\mu), \mu) \geq-N\}$ is a subset of $\left\{\mu: \sum_{i=1}^{n}\left|k_{i}\right| \leq N\right\}$, which is finite for any $N$. This establishes the conditions (8).

2.1.4. Remark. For $\mathfrak{g l}(n \mid n)$ one can interchange $\Delta^{\prime}$ and $\Delta^{\prime \prime}$ so the sum $\mathscr{F}_{T} T^{\prime \prime}(R)$ is well-defined. One readily sees that $\mathscr{F}_{T} T^{\prime \prime}(R)$ is not well-defined for $D(n+1 \mid n)$. For instance, for $n>1$, for each $k>0$ one has $v\left(-2 k \varepsilon_{1}\right)=0$ so $\max \operatorname{supp}\left(t_{-2 k \varepsilon_{1}}(R)\right)=0$ and the sum $\sum_{k=1}^{\infty} t_{-2 k \varepsilon_{1}}(R)$ is not well-defined; hence $\mathscr{F}_{T^{\prime \prime}}(R)$ is not well-defined as well.

2.2. By Section 1.3.3, $\hat{R}$ is an invertible element of $\mathscr{R}^{\prime}$. From representation theory we know that since $\hat{\mathfrak{g}}$ admits a Casimir element [Kac 1990, Chapter II], the character of the trivial $\hat{\mathfrak{g}}$-module is a linear combination of the characters of Verma $\hat{\mathfrak{g}}$-modules $M(\lambda)$, where $\lambda \in-\hat{Q}$ are such that $(\lambda+\hat{\rho}, \lambda+\hat{\rho})=(\hat{\rho}, \hat{\rho})$. Since the character of $M(\lambda)$ is equal to $\hat{R}^{-1} e^{\lambda}$, we obtain

$$
1=\sum_{\substack{\lambda \in \hat{Q}^{-} \\(\lambda+\hat{\rho}, \lambda+\hat{\rho})=(\hat{\rho}, \hat{\rho})}} a_{\lambda} \hat{R}^{-1} e^{\lambda},
$$

where $a_{\lambda} \in \mathbb{Z}$. This can be rewritten as

$$
\hat{R} e^{\hat{\rho}}=\sum_{\substack{\lambda \in \hat{\rho}-\hat{Q}^{+} \\(\lambda, \lambda)=(\hat{\rho}, \hat{\rho})}} a_{\lambda} e^{\lambda},
$$

that is $\operatorname{supp}(\hat{R}) \subset U$, see (6) for notation.

It remains to verify the inclusion $\operatorname{supp}\left(\mathscr{F} T^{\prime}\left(R e^{\hat{\rho}}\right)\right) \subset U$. The denominator identity for $\mathfrak{g}$ (see [Kac and Wakimoto 1994; Gorelik 2012]) takes the form

$$
\operatorname{Re}^{\rho}=\mathscr{F}_{W} W^{\prime \prime}\left(\frac{e^{\rho}}{\prod_{\beta \in S}\left(1+e^{-\beta}\right)}\right),
$$

where $S:=\left\{\varepsilon_{i}-\delta_{i}\right\}_{i=1}^{n}$ (the identity for $\mathfrak{g l}(n \mid n)$ immediately follows from the identity for $\mathfrak{s l}(n \mid n))$. Since $\rho=\hat{\rho}$ is $\hat{W}$-invariant, this implies

$$
t_{\mu}\left(R e^{\hat{\rho}}\right)=e^{\hat{\rho}} \sum_{w \in W^{\prime \prime}} \operatorname{sgn}(w) \prod_{\beta \in S}\left(1+e^{-t_{\mu} w \beta}\right)^{-1} .
$$


For each $t_{\mu} \in T^{\prime}$ and $w \in W^{\prime \prime}$ one has

$$
\operatorname{supp}\left(\prod_{\beta \in S}\left(1+e^{-t_{\mu} w \beta}\right)^{-1}\right) \subset V, \text { where } V:=\mathbb{Z}\left\{t_{\mu} w \beta: \beta \in S\right\} \cap \hat{Q}^{-} .
$$

Since $\left(t_{\mu} w \beta, t_{\mu} w \beta^{\prime}\right)=\left(\beta, \beta^{\prime}\right)=\left(t_{\mu} w \beta, \hat{\rho}\right)=(\hat{\rho}, \beta)=0$ for any $\beta, \beta^{\prime} \in S$, one has $(V, V)=(V, \hat{\rho})=0$. Therefore $V+\hat{\rho} \subset U$ so $\operatorname{supp}\left(t_{\mu}\left(R e^{\hat{\rho}}\right)\right) \subset U$ for each $\mu$. This establishes the required inclusion $\operatorname{supp}\left(\mathscr{F}_{T^{\prime}}\left(R e^{\hat{\rho}}\right)\right) \subset U$ and completes the proof of (5).

2.3. Let us deduce from (5) that the support of $\hat{R}^{-1} e^{\hat{\rho}} \cdot \mathscr{F}_{T} T^{\prime}\left(R e^{\hat{\rho}}\right)$ consists of $\hat{W}$ invariant elements of $\hat{Q}^{-}$. We do this in two steps: first, proving Lemma 2.3.1, which is valid for any simple contragredient Lie superalgebra and for $\mathfrak{g l}(n \mid n)$, and then, proving Proposition 2.3.2, which uses the fact that $\hat{\rho}=\rho$ for $\mathfrak{g}$ (this is equivalent to the fact that the dual Coxeter number is zero).

The affine root system $\hat{\Delta}^{\prime}$ is a subsystem of $\hat{\Delta}_{0}$. Set $\hat{\Delta}_{+}^{\prime}=\hat{\Delta}^{\prime} \cap \hat{\Delta}_{+}$and let $\hat{\Pi}^{\prime}$ be the corresponding set of simple roots. Fix $\hat{\rho}^{\prime} \in \hat{\mathfrak{h}}^{*}$ such that $2\left(\hat{\rho}^{\prime}, \alpha\right)=(\alpha, \alpha)$ for each $\alpha \in \hat{\Pi}^{\prime}$.

2.3.1. Lemma. The term $\hat{R}_{1} e^{\hat{\rho}^{\prime}-\hat{\rho}} \cdot \mathscr{F}^{\prime} T^{\prime}\left(R e^{\hat{\rho}}\right)$ is a $\hat{W}^{\prime}$-anti-invariant element of $\mathscr{R}_{\hat{W}^{\prime}}$.

Proof. By Section 2.1.1, $\mathscr{F}_{T^{\prime}}\left(R e^{\hat{\rho}}\right) \in \mathscr{R}$ and thus $\hat{R}_{1} e^{\hat{\rho}^{\prime}-\hat{\rho}} \cdot \mathscr{F}_{T} T^{\prime}\left(R e^{\hat{\rho}}\right) \in \mathscr{R}$.

Let $R_{0}^{\prime}, R_{0}^{\prime \prime}$ be the Weyl denominators for $\Delta^{\prime}, \Delta^{\prime \prime}$ (i.e., $R_{0}^{\prime}=\prod_{\alpha \in \Delta_{+}^{\prime}}\left(1-e^{-\alpha}\right)$ ). Notice that $R_{0}^{\prime \prime} e^{\hat{\rho}} / R_{1} \in \mathscr{R}^{\prime}$ so $w\left(R_{0}^{\prime \prime} e^{\hat{\rho}} / R_{1}\right)$ is well-defined. Below we will show that the sum $\mathscr{F}_{\hat{W}^{\prime}}\left(R_{0}^{\prime \prime} e^{\hat{\rho}} / R_{1}\right)$ is a well-defined element of $\mathscr{R}$ and will establish the following formula

$$
\mathscr{F}_{T^{\prime}}\left(R e^{\hat{\rho}}\right)=\mathscr{F}_{\hat{W^{\prime}}}\left(\frac{R_{0}^{\prime \prime} e^{\hat{\rho}}}{R_{1}}\right) .
$$

It is easy to see that $\hat{R}_{0} e^{\hat{\rho}^{\prime}}, \hat{R} e^{\hat{\rho}}$ are $\hat{W}^{\prime}$-anti-invariant elements of $\mathscr{R}^{\prime}$ (see, for instance, [Gorelik 2011, 1.5.1]). Since $\hat{R}_{1} e^{\hat{\rho}^{\prime}-\hat{\rho}} \in \mathscr{R}^{\prime}$ and $\hat{R}_{1} e^{\hat{\rho}^{\prime}-\hat{\rho}} \cdot \hat{R} e^{\hat{\rho}}=\hat{R}_{0} e^{\hat{\rho}^{\prime}}$, we conclude that $\hat{R}_{1} e^{\hat{\rho}^{\prime}-\hat{\rho}}$ is a $\hat{W}^{\prime}$-invariant element of $\mathscr{R}^{\prime}$. However, by Section 1.3.3, $\hat{R}_{1} \in \mathscr{R}_{\hat{W}}$, and thus $\hat{R}_{1} e^{\hat{\rho}^{\prime}-\hat{\rho}}$ is a $\hat{W}^{\prime}$-invariant element of $\mathscr{R}_{\hat{W}}$. Multiplying both sides of formula (9) by $\hat{R}_{1} e^{\hat{\rho}^{\prime}-\hat{\rho}}$ we obtain

$$
\hat{R}_{1} e^{\hat{\rho}^{\prime}-\hat{\rho}} \cdot \mathscr{F}_{T} T^{\prime}\left(R e^{\hat{\rho}}\right)=\mathscr{F}_{\hat{W}^{\prime}}\left(\frac{\hat{R}_{1}}{R_{1}} \cdot R_{0}^{\prime \prime} e^{\hat{\rho}^{\prime}}\right) .
$$

By Section 1.3.3, $\hat{R}_{1} / R_{1}$ and $R_{0}^{\prime \prime}$ lie in $\mathscr{R}_{\hat{W}}$. In the light of Section 1.3.5, the formula (10) implies the assertion of the lemma.

Let us show that the right-hand side of (9) is well-defined. Since $R_{0}^{\prime \prime}$ and $\hat{\rho}$ are $\hat{W}^{\prime}$-invariant, it is enough to check that $\mathscr{F}_{\hat{W}^{\prime}}\left(R_{1}^{-1}\right)$ is a well-defined element of $\mathscr{R}$. 
By Section 1.3.4, for each $w \in \hat{W}^{\prime}$ one has

$$
\max \operatorname{supp}\left(w\left(R_{1}^{-1}\right)\right)=\sum_{\substack{\beta \in \Delta_{1+}: \\ w \beta<0}} w \beta
$$

In particular, $\operatorname{supp}\left(w\left(R_{1}^{-1}\right)\right) \subset \hat{Q}^{-}$, so, if the term $\mathscr{F}_{\hat{W}^{\prime}}\left(R_{1}^{-1}\right)$ is well-defined, it lies in $\mathscr{R}$. In order to see that $\mathscr{F}_{\hat{W}^{\prime}}\left(R_{1}^{-1}\right)$ is well-defined let us check that for each $v \in \hat{Q}^{-}$the set

$$
X(v):=\left\{w \in \hat{W}^{\prime}: \sum_{\substack{\beta \in \Delta_{1+} \\ w \beta<0}} w \beta \geq v\right\}
$$

is finite. One has

$$
X(v) \subset\left\{w \in \hat{W}^{\prime}: w \beta \geq v \text { for all } \beta \in \Delta_{1+}\right\}
$$

Write $v=-k \delta+v^{\prime}$, where $k \geq 0, v^{\prime} \in Q$, and write $w \in X(v)$ in the form $w=t_{\mu} y$, where $t_{\mu} \in T^{\prime}, y \in W^{\prime}$. Since $w \beta=y \beta-(y \beta, \mu) \delta$ for $\beta \in \Delta_{1+}$, one has $(y \beta, \mu) \geq-k$ for each $\beta \in \Delta_{1+}$. Since $\left\{\varepsilon_{i}-\delta_{i}, \delta_{i}-\varepsilon_{i+1}\right\} \subset \Delta_{1+}$, this gives $\left|\left(\mu, y \delta_{i}\right)\right| \leq k$ for $i=1, \ldots, n$. Combining the facts that $W^{\prime}$ is a subgroup of signed permutation of $\left\{\delta_{j}\right\}_{j=1}^{n}$ and that $\left(\mu, \delta_{i}\right)$ is integral for each $i$, we conclude that $X(v)$ is finite. Thus $\mathscr{F}_{W^{\prime}}\left(R_{0}^{\prime \prime} e^{\hat{\rho}} / R_{1}\right)$ is a well-defined element of $\mathscr{R}$.

Now let us prove the formula (9). Recall that $\rho=\rho_{0}^{\prime}+\rho_{0}^{\prime \prime}-\rho_{1}$, where

$$
\rho_{0}^{\prime}:=\sum_{\alpha \in \Delta_{0+}^{\prime}} \alpha / 2, \quad \rho_{0}^{\prime \prime}:=\sum_{\alpha \in \Delta_{0+}^{\prime \prime}} \alpha / 2, \quad \rho_{1}:=\sum_{\beta \in \Delta_{1+}} \beta / 2
$$

The Weyl denominator identity for $\Delta_{0}^{\prime}$ takes the form

$$
R_{0}^{\prime} e^{\rho_{0}^{\prime}}=\mathscr{F}_{W^{\prime}}\left(e^{\rho_{0}^{\prime}}\right)
$$
Since $R_{1} e^{\rho_{1}}=\prod_{\beta \in \Delta_{1+}}\left(e^{\beta / 2}+e^{-\beta / 2}\right)$ is $W$-invariant and $R_{0}^{\prime \prime} e^{\rho_{0}^{\prime \prime}}$ is $W^{\prime}$-invariant, we
get

$$
R e^{\rho}=\frac{R_{0}^{\prime \prime} e^{\rho_{0}^{\prime \prime}}}{R_{1} e^{\rho_{1}}} \cdot \mathscr{F}_{W^{\prime}}\left(e^{\rho_{0}^{\prime}}\right)=\mathscr{F}_{W^{\prime}}\left(\frac{e^{\rho_{0}^{\prime}} R_{0}^{\prime \prime} e^{\rho_{0}^{\prime \prime}}}{R_{1} e^{\rho_{1}}}\right)=\mathscr{F}_{W^{\prime}}\left(\frac{R_{0}^{\prime \prime} e^{\rho}}{R_{1}}\right) .
$$

Using the $W$-invariance of $\hat{\rho}-\rho$, we obtain

$$
\mathscr{F}_{T^{\prime}}\left(R e^{\hat{\rho}}\right)=\mathscr{F}_{T^{\prime}}\left(\mathscr{F}_{W^{\prime}}\left(\frac{R_{0}^{\prime \prime} e^{\hat{\rho}}}{R_{1}}\right)\right)=\mathscr{F}_{W^{\prime}}\left(\frac{R_{0}^{\prime \prime} e^{\hat{\rho}}}{R_{1}}\right)
$$

as required. This completes the proof. 
2.3.2. Proposition. One has

$$
\operatorname{supp}\left(\hat{R}^{-1} e^{-\hat{\rho}} \cdot \mathscr{F}_{T^{\prime}}\left(\operatorname{Re}^{\hat{\rho}}\right)\right) \subset\left(\hat{Q}^{-}\right)^{\hat{W}}=\hat{Q}^{-} \cap \hat{Q}^{\perp} .
$$

Proof. Set

$$
Y:=\hat{R}^{-1} e^{-\hat{\rho}} \cdot \mathscr{F}_{T}\left(R e^{\hat{\rho}}\right) .
$$

By Sections 2.1.1 and 1.3.3, $\mathscr{F}^{\prime}\left(R e^{\hat{\rho}}\right), \hat{R}^{-1} \in \mathscr{R}$. Thus $Y \in \mathscr{R}$. One has

$$
\hat{R}_{0} e^{\hat{\rho}^{\prime}} Y=\hat{R}_{1} e^{\hat{\rho}^{\prime}-\hat{\rho}} \cdot \mathscr{F}_{T} T^{\prime}\left(R e^{\hat{\rho}}\right) .
$$

In the light of Lemma 2.3.1, we obtain

$$
\hat{R}_{0} e^{\hat{\rho}^{\prime}} Y \text { is a } \hat{W}^{\prime} \text {-anti-invariant element of } \mathscr{R}_{\hat{W}^{\prime}} \text {. }
$$

Write $Y=Y_{1}+Y_{2}$, where $\operatorname{supp}\left(Y_{1}\right)=\operatorname{supp}(Y) \cap \hat{Q}^{\perp}$ and $\operatorname{supp}\left(Y_{2}\right)=\operatorname{supp}(Y) \backslash \hat{Q}^{\perp}$. Note that $Y_{1}, Y_{2} \in \mathscr{R}$. Assume that $Y_{2} \neq 0$. Let $\mu$ be a maximal element in $\operatorname{supp}\left(Y_{2}\right)$. One has $\operatorname{supp}\left(\hat{R}^{-1}\right) \subset \hat{Q}^{-}$and $\operatorname{supp}\left(\mathscr{F}_{T^{\prime}}(R) e^{\hat{\rho}}\right) \subset \hat{\rho}-\hat{Q}^{+}$, by Section 1.3.4 and (5) respectively. Thus $\operatorname{supp}(Y) \subset \hat{Q}^{-}$and so $\mu \in \hat{Q}^{-}$.

Since $\operatorname{supp}\left(Y_{1}\right) \subset \hat{Q}^{\perp}, Y_{1}$ is a $\hat{W}$-invariant element of $\mathscr{R}_{\hat{W}}$. Recall that $\hat{R}_{0} e^{\hat{\rho}^{\prime}}$ is a $\hat{W}^{\prime}$-anti-invariant element of $\mathscr{R}_{\hat{W}}$. Thus $\hat{R}_{0} e^{\hat{\rho}^{\prime}} Y_{1}$ is a $\hat{W}^{\prime}$-anti-invariant element of $\mathscr{R}_{\hat{W}^{\prime}}$. In the light of (11), the product $\hat{R}_{0} e^{\hat{\rho}^{\prime}} Y_{2}$ is also a $\hat{W}^{\prime}$-anti-invariant element of $\mathscr{R}_{\hat{W}^{\prime}}$. Clearly, $\hat{\rho}^{\prime}+\mu$ is a maximal element in the support of $\hat{R}_{0} e^{\hat{\rho}^{\prime}} Y_{2}$. By Section 1.3.5, this support is a union of $\hat{W}^{\prime}$-regular orbits (recall that regularity means that each element has the trivial stabilizer in $\left.\hat{W}^{\prime}\right)$, so $\hat{\rho}^{\prime}+\mu$ is a maximal element in a regular $\hat{W}^{\prime}$-orbit and thus $2\left(\hat{\rho}^{\prime}+\mu, \alpha\right) /(\alpha, \alpha) \notin \mathbb{Z}_{\leq 0}$ for each $\alpha \in \hat{\Pi}^{\prime}$. Since $\mu \in \hat{Q}^{-}$one has $2(\mu, \alpha) /(\alpha, \alpha) \in \mathbb{Z}$ for each $\alpha \in \hat{\Pi}^{\prime}$. Taking into account that $2\left(\hat{\rho}^{\prime}, \alpha\right) /(\alpha, \alpha)=1$ for each $\alpha \in \hat{\Pi}^{\prime}$, we obtain

$$
\frac{2(\mu, \alpha)}{(\alpha, \alpha)} \in \mathbb{Z}_{\geq 0} \quad \text { for all } \alpha \in \hat{\Pi}^{\prime}
$$

Recall that $\delta=\sum_{\alpha \in \hat{\Pi}^{\prime}} k_{\alpha} \alpha$ for some $k_{\alpha} \in \mathbb{Z}_{>0}$ (see [Kac 1990, Chapter VI]). Since $\mu \in \hat{Q}^{-}$one has $(\mu, \delta)=0$. Combining with (12), we get $(\mu, \alpha)=0$ for each $\alpha \in \hat{\Pi}^{\prime}$ so $\mu \in\left(\hat{\Delta}^{\prime}\right)^{\perp}$.

Let us show that $(\mu, \mu)=0$. Since $(\hat{\rho}, \hat{Q})=0$, it is equivalent to the equality $(\mu+\hat{\rho}, \mu+\hat{\rho})=(\hat{\rho}, \hat{\rho})$. Notice that $\mu+\hat{\rho}$ is a maximal element in the support of $\hat{R} e^{\hat{\rho}} Y_{2}$. Let us check that

$$
\operatorname{supp}\left(\hat{R} e^{\hat{\rho}} Y_{2}\right) \subset U=\left\{\xi \in \hat{\rho}-\hat{Q}^{+}:(\xi, \xi)=(\hat{\rho}, \hat{\rho})\right\} .
$$

Indeed,

$$
\hat{R} e^{\hat{\rho}} Y_{2}=\mathscr{F}_{T^{\prime}}\left(\operatorname{Re}^{\hat{\rho}}\right)-\hat{R} e^{\hat{\rho}} Y_{1}
$$

and, by (5),

$$
\operatorname{supp}\left(\mathscr{F}_{T^{\prime}}\left(R e^{\hat{\rho}}\right)\right) \subset U \quad \text { and } \quad \operatorname{supp}\left(\hat{R} e^{\hat{\rho}}\right) \subset U .
$$


By construction, $\operatorname{supp}\left(Y_{1}\right) \subset \hat{Q}^{\perp} \cap \hat{Q}^{-}$. Recall that $\hat{\rho}=\rho \in \mathbb{Q} \Delta$, so $U \subset \mathbb{Q} \cdot \hat{Q}$. In particular, we have $\left(U, \operatorname{supp}\left(Y_{1}\right)\right)=0$. Since $\left(\operatorname{supp}\left(Y_{1}\right), \operatorname{supp}\left(Y_{1}\right)\right)=0$, we obtain $\left(\operatorname{supp}\left(Y_{1}\right)+U\right) \subset U$ and this establishes the inclusion $(13)$. Hence $(\mu, \mu)=0$.

Recall that $\mu \in\left(\hat{\Delta}^{\prime}\right)^{\perp} \cap \hat{Q}^{-}$. One has

$$
\left(\hat{\Delta}^{\prime}\right)^{\perp} \cap \hat{Q}=\left(\hat{Q}^{\perp} \cap \hat{Q}\right) \oplus \mathbb{Z} \Delta^{\prime \prime} .
$$

For every $\beta \in \hat{Q}^{\perp} \cap \hat{Q}, \gamma \in \Delta^{\prime \prime}$ one has $(\beta, \beta)=(\beta, \gamma)=0$ and $(\gamma, \gamma) \neq 0$ if $\gamma \neq 0$. Using the equality $(\mu, \mu)=0$, we get $\mu \in \hat{Q}^{\perp} \cap \hat{Q}$, which contradicts to the construction of $Y_{2}$. Hence $Y_{2}=0$ as required.

2.3.3. Corollary. For $\mathfrak{g}=D(n+1 \mid n)$ one has $f(q) \cdot \hat{R} e^{\hat{\rho}}=\mathscr{F}_{T^{\prime}}\left(\operatorname{Re} e^{\hat{\rho}}\right)$ for some $f(q)=\sum_{k=0}^{\infty} a_{k} q^{k}\left(a_{k} \in \mathbb{Z}\right)$. For $\mathfrak{g}=\mathfrak{g l}(n \mid n)$ one has $f\left(q, e^{\mathfrak{s t r}}\right) \cdot \hat{\operatorname{R}} e^{\hat{\rho}}=\mathscr{F}_{T} T^{\prime}\left(\operatorname{Re}^{\hat{\rho}}\right)$ for some $f\left(q, e^{\mathfrak{s t r}}\right)=\sum_{k=0}^{\infty} \sum_{m=-\infty}^{\infty} a_{k, m} q^{k} e^{m \cdot \mathfrak{s t r}}\left(a_{k, m} \in \mathbb{Z}\right)$.

Proof. One has $(\hat{Q})^{\perp} \cap \hat{Q}=\mathbb{Z} \delta+\mathbb{Z} \mathfrak{s t r}$ for $\mathfrak{g l}(n \mid n)$ and $(\hat{Q})^{\perp} \cap \hat{Q}=\mathbb{Z} \delta$ for $D(n+1 \mid n)$.

2.4. In this subsection we complete the proof of the denominator identities (2) by proving the formulas (3). We prove them by taking a suitable evaluation of the term $\hat{R}^{-1} e^{-\hat{\rho} \mathscr{F}} T^{\prime}\left(R e^{\hat{\rho}}\right)$. Since $\hat{\rho}$ is $\hat{W}$-invariant, this term is equal to $\hat{R}^{-1} \mathscr{F}_{F} T^{\prime}(R)$, and, by Corollary 2.3.3, it is equal to $f(q)$ for $D(n+1 \mid n)$ and to $f\left(q, e^{\mathfrak{s t r}}\right)$ for $\mathfrak{g l}(n \mid n)$. Now we consider $q$ as a real parameter between 0 and 1 . We choose the evaluation in such a way that the evaluation of $\hat{R}^{-1} \mathscr{F}_{T^{\prime}}(R)=\hat{R}^{-1} \sum_{t \in T^{\prime}} t(R)$ is equal to the evaluation of $\hat{R}^{-1} R$. As a result, $f(q)$ (resp., $f\left(q, e^{\mathfrak{s t r}}\right)$ ) is equal to the evaluation of $\hat{R}^{-1} R$, which can be easily computed.

2.4.1. Case $D(n+1 \mid n)$. Take a complex parameter $x$ and consider the evaluation $e^{-\varepsilon_{i}}:=x^{a_{i}}, e^{-\delta_{j}}:=-x^{b_{j}}$, where $a_{i}(i=1, \ldots, n+1)$ and $b_{j}(j=1, \ldots, n)$ are integers such that $a_{i} \pm b_{j} \neq 0, a_{i} \pm a_{j} \neq 0, b_{i} \pm b_{j} \neq 0, b_{i} \neq 0$ for all indexes $i, j$. We denote by $\hat{R}$ and $\hat{R}(x)$ the evaluation of $R$ and $R(x)$. The functions $R(x)$ and $\hat{R}(x)$ are meromorphic. One has

$$
R(x)=\frac{\prod_{1 \leq i<j \leq n+1}\left(1-x^{a_{i} \pm a_{j}}\right) \cdot \prod_{1 \leq i<j \leq n}\left(1-x^{b_{i} \pm b_{j}}\right) \cdot \prod_{1 \leq i \leq n}\left(1-x^{2 b_{i}}\right)}{\prod_{1 \leq i \leq j \leq n}\left(1-x^{a_{i} \pm b_{j}}\right) \prod_{1 \leq j<i \leq n+1}\left(1-x^{b_{j} \pm a_{i}}\right)} .
$$

One readily sees that $R(x)$ has a pole at $x=1$ of order $\left|\Delta_{1+}\right|-\left|\Delta_{0+}\right|=n$.

One has

$$
\left.\frac{\hat{R}(x)}{R(x)}\right|_{x=1}=\frac{\left((1-q)_{q}^{\infty}\right)^{\operatorname{dim} \mathfrak{g}_{0}}}{\left((1-q)_{q}^{\infty}\right)^{\operatorname{dim} \mathfrak{g}_{1}}}=\left((1-q)_{q}^{\infty}\right)^{\operatorname{dim} \mathfrak{g}_{0}-\operatorname{dim} \mathfrak{g}_{1}}=(1-q)_{q}^{\infty} .
$$

In particular, $\hat{R}(x)$ also has a pole of order $n$ at $x=1$. 
The evaluation of $\left(t_{\sum k_{i} \delta_{i}}(R)\right)(x)$ is

$$
\frac{\prod_{1 \leq i<j \leq n+1}\left(1-x^{a_{i} \pm a_{j}}\right) \cdot \prod_{1 \leq i \leq n}\left(1-q^{-2 k_{i}} x^{2 b_{i}}\right) \cdot \prod_{1 \leq i<j \leq n}\left(1-q^{-k_{i} \mp k_{j}} x^{b_{i} \pm b_{j}}\right)}{\prod_{1 \leq i \leq j \leq n}\left(1-q^{\mp k_{j}} x^{a_{i} \pm b_{j}}\right) \prod_{1 \leq j<i \leq n+1}\left(1-q^{-k_{j}} x^{b_{j} \pm a_{i}}\right)}
$$

which is a meromorphic function. Let $s$ be the number of zeros among $k_{1}, \ldots, k_{n}$. Then at $x=1$ the order of zero of the numerator is at least is $n(n+1)+s^{2}$, and the order of zero of the denominator is $2(n+1) s$. Therefore at $x=1$ the function $\left(t_{\sum k_{i} \delta_{i}}(R)\right)(x)$ has the pole of order at most $2(n+1) s-n(n+1)-s^{2}=$ $n+1-(n+1-s)^{2}$; in particular, $\left(t_{\sum k_{i} \delta_{i}}(R)\right)(x)$ has the pole of order at most $n$ and it is equal to $n$ if and only if $n=s$ that is $\sum k_{i} \delta_{i}=0$ and $\left(t_{\sum k_{i} \delta_{i}}(R)\right)(x)=R(x)$.

We conclude that

$$
(\hat{R}(x))^{-1} \cdot \sum_{t \in T^{\prime}: t \neq \mathrm{id}}(t(R))(x)
$$

is holomorphic at $x=1$ and its value is zero, and that

$$
(\hat{R}(x))^{-1} \cdot \sum_{t \in T^{\prime}}(t(R))(x)
$$
is holomorphic at $x=1$ and its value is $\left.\frac{R(x)}{\hat{R}(x)}\right|_{x=1}$. In the light of Corollary 2.3.3
we obtain

$$
f(q)=\left.\frac{R(x)}{\hat{R}(x)}\right|_{x=1}=\left((1-q)_{q}^{\infty}\right)^{-1} .
$$

2.4.2. Case $\mathfrak{g l}(n \mid n)$. Fix $y>1$. Take a complex parameter $x$ and consider the following evaluation

$$
e^{-\varepsilon_{1}}:=y, e^{-\varepsilon_{i}}:=x^{i}, \text { for } i=2, \ldots, n ; e^{-\delta_{i}}:=-x^{-i} \text { for } i=1, \ldots, n .
$$

The functions $R(x), \hat{R}(x)$ are meromorphic. One has

$$
R(x)=\frac{\prod_{1<i \leq n}\left(1-y x^{-i}\right) \cdot \prod_{1<i<j \leq n}\left(1-x^{i-j}\right) \cdot \prod_{1 \leq i<j \leq n}\left(1-x^{j-i}\right)}{\prod_{1 \leq i \leq n}\left(1-y x^{i}\right) \cdot \prod_{1<i \leq j \leq n}\left(1-x^{i+j}\right) \cdot \prod_{1 \leq j<i \leq n}\left(1-x^{-i-j}\right)} .
$$

Therefore the function $R(x)$ has a pole of order $n-1$ at $x=1$.

One has

$$
\left.\frac{\hat{R}(x)}{R(x)}\right|_{x=1}=\frac{\left((1-q)_{q}^{\infty}\right)^{\operatorname{dim} \mathfrak{g}_{0}-2(n-1)} \cdot\left((1-q y)_{q}^{\infty}\right)^{n-1} \cdot\left(\left(1-q y^{-1}\right)_{q}^{\infty}\right)^{n-1}}{\left((1-q)_{q}^{\infty}\right)^{\operatorname{dim} \mathfrak{g}_{1}-2 n} \cdot\left((1-q y)_{q}^{\infty}\right)^{n} \cdot\left(\left(1-q y^{-1}\right)_{q}^{\infty}\right)^{n}} .
$$

Thus $\hat{R}(x)$ also has a pole of order $n-1$ at $x=1$. Since $\operatorname{dim} \mathfrak{g}_{0}=\operatorname{dim} \mathfrak{g}_{1}$ and $e^{\mathfrak{s t r}}=(-1)^{n} y^{-1}$ for $x=1$ we obtain

$$
\left.\frac{\hat{R}(x)}{R(x)}\right|_{x=1}=\frac{\left((1-q)_{q}^{\infty}\right)^{2}}{\left(1-q(-1)^{n} e^{\mathfrak{s t r}}\right)_{q}^{\infty} \cdot\left(1-q(-1)^{n} e^{-\mathfrak{s t r}}\right)_{q}^{\infty}} .
$$


One has

$\left(t_{\sum k_{i} \delta_{i}}(R)\right)(x, y)$

$$
=\frac{\prod_{1<i \leq n}\left(1-y x^{-i}\right) \cdot \prod_{1<i<j \leq n}\left(1-x^{i-j}\right) \cdot \prod_{1 \leq i<j \leq n}\left(1-q^{k_{j}-k_{i}} x^{j-i}\right)}{\prod_{1 \leq i \leq n}\left(1-q^{k_{i}} y x^{i}\right) \cdot \prod_{1<i \leq j \leq n}\left(1-q^{k_{j}} x^{i+j}\right) \cdot \prod_{1 \leq j<i \leq n}\left(1-q^{-k_{j}} x^{-i-j}\right)},
$$

which is a meromorphic function.

Let $s$ be the number of zeros among $k_{1}, \ldots, k_{n}$. Then at $x=1$ the order of zero of the numerator is at least

$$
\frac{(n-1)(n-2)+s(s-1)}{2},
$$

and the order of zero of the denominator is $(n-1) s$. Therefore at $x=1$ the function $\left(t_{\sum k_{i} \delta_{i}}(R)\right)(x, y)$ has a pole of order at most

$$
(n-1) s-\frac{(n-1)(n-2)+s(s-1)}{2}=\frac{3 n-s-2-(n-s)^{2}}{2},
$$

so the order is at most $n-1$ and it is equal to $n-1$ if and only if $s=n-1, n$. Notice that $s \neq n-1$, since $\sum k_{i}=0$. Therefore the pole has order $n-1$ if and only if $\sum k_{i} \delta_{i}=0$.

We conclude that the function $(\hat{R}(x))^{-1}\left(\mathscr{F}_{T^{\prime}}(R)\right)(x)$ is holomorphic at $x=1$ and its value is $\left.(R(x) / \hat{R}(x))\right|_{x=1}$. Using Corollary 2.3.3 we obtain

$$
f\left(q, e^{\mathfrak{s t r}}\right)=\left.\frac{R(x)}{\hat{R}(x)}\right|_{x=1}=\frac{\left(1-q(-1)^{n} e^{\mathfrak{s t r}}\right)_{q}^{\infty} \cdot\left(1-q(-1)^{n} e^{-\mathfrak{s t r}}\right)_{q}^{\infty}}{\left((1-q)_{q}^{\infty}\right)^{2}}
$$

\section{Other forms of denominator identity}

Recall that the denominator identity for a basic Lie superalgebra can be written in the form

$$
\operatorname{Re}^{\rho}=\mathscr{F}_{W^{\sharp}}\left(\frac{e^{\rho}}{\prod_{\beta \in S}\left(1+e^{-\beta}\right)}\right),
$$

where $S \subset \Pi$ is the maximal isotropic system, and $W^{\sharp}$ is the Weyl group of the "largest" root subsystem of $\Delta_{0}\left(\Delta_{0}=\Delta^{\prime} \amalg \Delta^{\prime \prime}\right)$, see [Kac and Wakimoto 1994; Gorelik 2012]; in particular, $W^{\sharp}:=W^{\prime \prime}$ for $\mathfrak{g}=D(n+1 \mid n)$, and $W^{\sharp}:=W^{\prime}$ or $W^{\sharp}:=W^{\prime \prime}$ for $\mathfrak{g}=\mathfrak{g l}(n \mid n)$.

If the dual Coxeter number of $\mathfrak{g}$ is nonzero the affine denominator identity for $\mathfrak{g}$ can be written in the form

$$
\hat{R} e^{\hat{\rho}}=\mathscr{F}_{W^{\sharp}}\left(\frac{e^{\hat{\rho}}}{\prod_{\beta \in S}\left(1+e^{-\beta}\right)}\right),
$$


see [Gorelik 2012, 2.1]. In this section we will show that for $\mathfrak{g l}(n \mid n)$ the denominator identity can be written in a similar form:

$$
\hat{R} e^{\rho}=f\left(q, e^{\mathfrak{s t r}}\right) \cdot \mathscr{F}_{\hat{W}^{\prime}}\left(\frac{e^{\rho}}{\prod_{\beta \in S}\left(1+e^{-\beta}\right)}\right),
$$

and that the denominator identities for $D(n+1 \mid n)$ can not be written in a similar form, since the expressions

$$
\mathscr{F}_{\hat{W}^{\prime \prime}}\left(\frac{e^{\rho}}{\prod_{\beta \in S}\left(1+e^{-\beta}\right)}\right) \text { and } \mathscr{F}_{\hat{W}^{\prime}}\left(\frac{e^{\rho}}{\prod_{\beta \in S}\left(1+e^{-\beta}\right)}\right)
$$

are not well-defined.

3.1. Case $D(n+1 \mid n)$. Let us show that the expressions in (16) are not well-defined for $D(n+1 \mid n)$. Fix $\Pi$ as in Section 1.1 and recall that $\rho=0$.

We repeat the reasoning of Section 2.1.1. One has

$$
\sum_{\beta \in V_{S}(w)} w \beta \in \operatorname{supp}\left(\frac{1}{\prod_{\beta \in S}\left(1+e^{-w \beta}\right)}\right) \subset \sum_{\beta \in V_{S}(w)} w \beta-\hat{Q}^{+} \subset \hat{Q}^{-},
$$

where

$$
V_{S}(w)=\{\beta \in S: w \beta<0\} .
$$

Therefore $1 \in \operatorname{supp}\left(1 / \prod_{\beta \in S}\left(1+e^{-w \beta}\right)\right)$ if and only if $w S \subset \Delta_{+}$.

Take $S=\left\{\varepsilon_{i}-\delta_{i}\right\}$; then $t_{\mu} S \subset \Delta_{+}$if $\left(\varepsilon_{i}-\delta_{i}, \mu\right)<0$ for all $i$ which holds for all $\mu \in \sum \mathbb{Z}_{<0} \varepsilon_{i}$ and all $\mu \in \sum \mathbb{Z}_{>0} \delta_{i}$. Hence the sums in (16) contain infinitely many summands equal to 1 and thus they are not well-defined.

3.2. Case $\mathfrak{g l}(\boldsymbol{n} \mid \boldsymbol{n})$. Fix $\Pi$ as in Section 1.1 ; then $S=\left\{\varepsilon_{i}-\delta_{i}\right\}$.

In order to deduce the formula (15) from (14) and (2) it is enough to verify that the expression

$$
\mathscr{\mathscr { F }}_{\hat{W}^{\prime}}\left(\frac{e^{\rho}}{\prod_{\beta \in S}\left(1+e^{-\beta}\right)}\right)=e^{\rho \mathscr{F}_{\hat{W}}} \hat{W}^{\prime}\left(\frac{1}{\prod_{\beta \in S}\left(1+e^{-\beta}\right)}\right)
$$

is well-defined (since $\rho$ is $\hat{W}$-invariant). As in Section 2.1.1, this amounts to showing that

$$
X_{S}(v):=\left\{w \in \hat{W}^{\prime}: \sum_{\beta \in V_{S}(w)} w \beta \geq-v\right\}
$$

is finite for any $v \in \hat{Q}^{+}$(where $V_{S}(w)$ is defined as in Section 3.1). As in Section 2.1.1, writing $v=k \delta+v_{+}$, where $v_{+} \in \mathbb{Z} \Delta$, we get

$$
X_{S}(\nu) \subset\left\{t_{\mu} y: \mu \in T^{\prime}, y \in W^{\prime} \text { s.t. }(y \beta, \mu) \geq-k \text { for all } \beta \in S\right\} .
$$


Since $y$ permutes $\delta_{i} \mathrm{~s}, t_{\mu} y \in X_{S}(v)$ forces $\left(\delta_{i}, \mu\right) \geq-k$ for all $i$. Taking into account that $\mu$ lies in the $\mathbb{Z}$-span of $\delta_{i}$ and $\left(\mu, \sum_{i=1}^{n} \delta_{i}\right)=0$, we conclude that $X_{S}(v)$ is finite. This establishes (15).

\section{Acknowledgement}

A part of this manuscript was written during the first author's stay at Max Planck Institut für Mathematik in Bonn, whose hospitality is greatly appreciated. The authors are grateful to P. Etingof and to V. Kac for fruitful discussions.

\section{References}

[De Sole and Kac 2005] A. De Sole and V. G. Kac, "On integral representations of $q$-gamma and q-beta functions", Rend. Lincei Mat. Appl. 16:1 (2005), 11-29. MR 2007a:33013 Zbl 1225.33017

[Gorelik 2010] M. Gorelik, "Weyl denominator identity for affine Lie superalgebra $\mathfrak{g l}(2 \mid 2)$ ", preprint, 2010. arXiv 1007.4305v1

[Gorelik 2011] M. Gorelik, "Weyl denominator identity for affine Lie superalgebras with non-zero dual Coxeter number”, J. Algebra 337 (2011), 50-62. MR 2012c:17038 Zbl 05996430

[Gorelik 2012] M. Gorelik, "Weyl denominator identity for finite-dimensional Lie superalgebras", pp. 167-188 in Highlights in Lie algebraic methods (Bremen, 2009), edited by A. Joseph et al., Progr. Math. 295, Birkhäuser, New York, 2012. MR 2866851

[Jacobi 1829] C. Jacobi, Fundamenta nova theoriae functionum ellipticarum, Regiomonti Sumptibus Fratrum Bornträger, 1829. Reprinted in Gesammelte Werke, Erster Band, pp. 55-239, Reimer, 1881.

[Kac 1977] V. G. Kac, "Lie superalgebras”, Advances in Math. 26:1 (1977), 8-96. MR 58 \#5803 Zbl 0366.17012

[Kac 1990] V. G. Kac, Infinite-dimensional Lie algebras, 3rd ed., Cambridge University Press, 1990. MR 92k:17038 Zbl 0716.17022

[Kac and Wakimoto 1994] V. G. Kac and M. Wakimoto, "Integrable highest weight modules over affine superalgebras and number theory", pp. 415-456 in Lie theory and geometry, edited by J.-L. Brylinski et al., Progr. Math. 123, Birkhäuser, Boston, MA, 1994. MR 96j:11056 Zbl 0854.17028

[Zagier 2000] D. Zagier, "A proof of the Kac-Wakimoto affine denominator formula for the strange series”, Math. Res. Lett. 7:5 (2000), 597-604. MR 2001m:11064 Zbl 1125.11319

Communicated by Georgia Benkart

Received 2010-12-13 Revised 2011-04-09 Accepted 2011-08-14

maria.gorelik@weizmann.ac.il Department of Mathematics, Weizmann Institute of Science, POB 26, 76100 Rehovot, Israel http://www.wisdom.weizmann.ac.il/ gorelik/

shifi@umich.edu

Department of Mathematics, University of Michigan, 530 Church Street, Ann Arbor, MI 48109, United States 


\section{Algebra \& Number Theory}

msp.berkeley.edu/ant

\section{EDITORS}

MANAGING EDITOR

Bjorn Poonen

Massachusetts Institute of Technology

Cambridge, USA

\author{
EDITORIAL BOARD CHAIR \\ David Eisenbud \\ University of California \\ Berkeley, USA
}

\section{BOARD OF EDITORS}

Georgia Benkart

Dave Benson

Richard E. Borcherds

John H. Coates

J-L. Colliot-Thélène

Brian D. Conrad

Hélène Esnault

Hubert Flenner

Edward Frenkel

Andrew Granville

Joseph Gubeladze

Ehud Hrushovski

Craig Huneke

Mikhail Kapranov

Yujiro Kawamata

János Kollár

Yuri Manin

Barry Mazur

Philippe Michel
University of Wisconsin, Madison, USA

University of Aberdeen, Scotland

University of California, Berkeley, USA

University of Cambridge, UK

CNRS, Université Paris-Sud, France

University of Michigan, USA

Universität Duisburg-Essen, Germany

Ruhr-Universität, Germany

University of California, Berkeley, USA

Université de Montréal, Canada

San Francisco State University, USA

Hebrew University, Israel

University of Kansas, USA

Yale University, USA

University of Tokyo, Japan

Princeton University, USA

Northwestern University, USA

Harvard University, USA

École Polytechnique Fédérale de Lausanne
Susan Montgomery

Shigefumi Mori

Raman Parimala

Jonathan Pila

Victor Reiner

Karl Rubin

Peter Sarnak

Joseph H. Silverman

Michael Singer

Vasudevan Srinivas

J. Toby Stafford

Bernd Sturmfels

Richard Taylor

Ravi Vakil

Michel van den Bergh

Marie-France Vignéras

Kei-Ichi Watanabe

Andrei Zelevinsky

Efim Zelmanov
University of Southern California, USA

RIMS, Kyoto University, Japan

Emory University, USA

University of Oxford, UK

University of Minnesota, USA

University of California, Irvine, USA

Princeton University, USA

Brown University, USA

North Carolina State University, USA

Tata Inst. of Fund. Research, India

University of Michigan, USA

University of California, Berkeley, USA

Harvard University, USA

Stanford University, USA

Hasselt University, Belgium

Université Paris VII, France

Nihon University, Japan

Northeastern University, USA

University of California, San Diego, USA

\section{PRODUCTION}

contact@msp.org

Silvio Levy, Scientific Editor

See inside back cover or www.jant.org for submission instructions.

The subscription price for 2012 is US \$175/year for the electronic version, and \$275/year (+ \$40 shipping outside the US) for print and electronic. Subscriptions, requests for back issues from the last three years and changes of subscribers address should be sent to Mathematical Sciences Publishers, Department of Mathematics, University of California, Berkeley, CA 94720-3840, USA.

Algebra \& Number Theory (ISSN 1937-0652) at Mathematical Sciences Publishers, Department of Mathematics, University of California, Berkeley, CA 94720-3840 is published continuously online. Periodical rate postage paid at Berkeley, CA 94704, and additional mailing offices.

ANT peer review and production are managed by EditFLOW ${ }^{\circledR}$ from Mathematical Sciences Publishers.

PUBLISHED BY

mathematical sciences publishers

http://msp.org/

A NON-PROFIT CORPORATION

Typeset in IATEX

Copyright $@ 2012$ by Mathematical Sciences Publishers 


\section{Algebra \& Number Theory}

Volume $6 \quad$ No. $5 \quad 2012$

Fields of moduli of three-point $G$-covers with cyclic $p$-Sylow, I

ANDREW OBUS

Toroidal compactifications of PEL-type Kuga families

KAI-WEN LAN

Idempotents in representation rings of quivers

RYAN KINSER and RALF SCHIFFLER

Cox rings and pseudoeffective cones of projectivized toric vector bundles

José González, Milena Hering, Sam Payne and Hendrik SÜss

Squareful numbers in hyperplanes

KARL VAN VALCKENBORGH

A denominator identity for affine Lie superalgebras with zero dual Coxeter number MARIA GORELIK and SHIFRA REIF 